\title{
A METHOD FOR SPECIFYING SEMANTICS OF LARGE SETS OF 3D MODELS
}

\author{
Xin Zhang ${ }^{1}$, Tim Tutenel ${ }^{2}$, Rong Mo ${ }^{1}$, Rafael Bidarra ${ }^{2}$ and Willem F. Bronsvoort ${ }^{2}$ \\ ${ }^{l}$ Northwestern Polytechnical University, China \\ zhangx0325@hotmail.com,morong@nwpu.edu.cn \\ ${ }^{2}$ Computer Graphics Group, Delft University of Technology, the Netherlands \\ tim.tutenel@gmail.com, \{r.bidarra,w.f.bronsvoort\}@tudelft.nl
}

Keywords: model classification; segmentation; annotation; semantics

Abstract: $\quad$ Semantics of 3D models is playing a crucial role in games and simulations. In this paper we propose a framework to specify semantics of large sets of 3D models with minimal human involvement. The framework consists of three modules: classification, segmentation and annotation. We associate a few models with tags representing their classes and classify the other models automatically. Once all models have been classified in different groups, we take a certain number of models as template models in each group, and segment these template models interactively. We then use the segmentation method (and parameters) of the template models to segment the rest of the models of the same group automatically. We annotate the interactively segmented parts and use an attributed adjacency graph to represent them. Automatic annotation of the rest of the models is then performed by subgraph matching. Experiments show that the proposed framework can effectively specify semantics of large sets of 3D models.

\section{INTRODUCTION}

With rapid advancements in modeling techniques and graphics hardware, very complex and visually convincing 3D models become more important in virtual worlds. In recent years, quite some research has been done on semantics of models, to make them behave more as in real-life. Semantics of models is all information about the model, in addition to its geometry (Bronsvoort et al., 2010). This can include various parameters, e.g. for physical properties, roles, behavior and services they provide. Semantic information of models can be useful in many applications: 1) by using semantic models when generating virtual worlds automatically, the output can be improved (for example, information about placement relationships can create more realistic layouts (Tutenel et al., 2009)); 2) detailed and extensive information about object interaction, and services provided by objects, can lead to more immersive and compelling gameplay experiences (Kessing et al., 2009); and 3) behavioral information can be used to maintain semantic consistency in adaptive environments.
More on the role semantics can play in virtual worlds can be found in (Tutenel et al., 2008).

However, specifying semantics of 3D models is often a laborious and repetitive task. It is therefore important to find techniques to assist a user in this task. Semantics of 3D models can generally be divided into two categories: global semantics and local semantics. Global semantics describes general information about 3D models, including their names, materials, volumes and relationships with other models. Local semantics describes information on the individual parts of which the model is composed, and relationships among these parts. To our knowledge, most current methods specify semantics of 3D models manually or semi-automatically, and usually the semi-automatic methods still require a great deal of user interaction. Our method tries to reduce the amount of interaction. For specifying global semantics, some methods propose to classify the 3D models and use the semantics of already classified models to annotate the unknown ones if they are in same group. However, due to the mismatch between the high-level human intention and the low-level geometric data representation, usually called the "semantic gap" (Smeulder et al., 2000), the results of automatic classification are not 
convincing. For specifying local semantics, we need to segment 3D models into meaningful parts and annotate each part. The problem of segmentation of $3 \mathrm{D}$ models is still a challenge, because current methods cannot automatically separate models into meaningful parts without context.

In this paper, we propose a method for specifying global and local semantics of 3D models with minimal human intervention. We manually annotate a few models with tags (global semantics) as training models. We classify the rest of the models based on these training models and associate them with the tag of the training models in the same class. After all the models have been classified, we choose a few models as template models from each class. The template models are segmented and the model parts are annotated with tags (local semantics) interactively. The segmentation method (and parameters) of template models can be used to partition the models with the same tag. Once the models have been separated into parts, we rely on Attributed Adjacency Graph (AAG) to represent the model parts. The AAG representing the parts of a template model contains local semantics. Therefore, specifying the local semantics of partitioned models can be accomplished by subgraph isomorphism. The main contributions of this paper include: 1) A framework for specifying semantics of 3D models with limited human involvement; 2) The use of subgraph isomorphism for annotation of model parts.

The remainder of this paper is organized as follows. In Section 2, we introduce the related work on specifying semantics of 3D models. The outline of our method is described in Section 3. Specifying local semantics of 3D models is described in Section 4. Our prototype system is presented in Section 5, and we show results and give conclusions in Sections 6 and 7.

\section{RELATED WORK}

In order to classify 3D models, we need to define a shape descriptor first, which is used to compute the similarity between 3D models. In the past decade, many shape descriptors have been proposed in the scope of shape-based 3D model retrieval. Some shape descriptors rely on geometry to compare 3D models, such as Shape distribution (Osada et al., 2002), Spherical harmonic (Saupe and Vranic, 2001) and 3D Zernike (Novotni and Klein, 2004)). Reeb graph (Biasotti et al., 2008) is based on topology. A more general overview of shape descriptor can be found in the survey (Tangelder and Veltkamp,
2004). Despite of years of research, the retrieval performances of above-mentioned methods are very limited. Once the shape descriptor for computing the similarity between models has been determined, we can resort to several methods to classify the models, such K-NN (Han and Karypis, 2000), SVM (Novotni et al., 2005), and neural networks (Carpenter and Hoffman, 1997).

All approaches described above use geometry or topology to compute the similarity between 3D models. Actually, some models are similar in shape, but they are not similar at all when looking at their meaning, because of the semantic gap. For example, in Fig. 1, the models "Bottle" and "Balloon vehicle" are similar in shape, but they are quite different objects. Relevance feedback is often used to bridge the semantic gap. The most important part of relevance feedback is the learning algorithm. Some approaches (Rocchio, 1971) modify query vectors or change the weight of elements of the vector to make it move towards positive and away from negative examples. Other methods (Giacinto and Roli, 2004) use a Bayesian framework to estimate the posteriori probability after each iteration. Recently, several kinds of SVM (Novotni et al., 2005) with different kernels have been successfully utilized in classification and relevance feedback due to its efficiency and precision.
Bottle

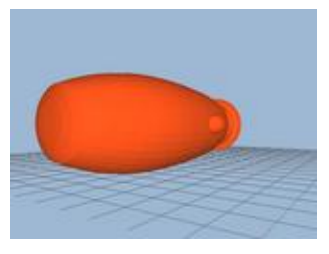

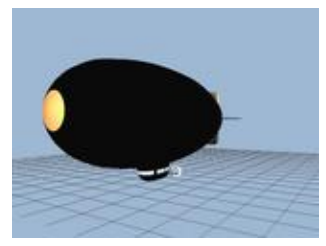

Balloon vehicle
Fig. 1. 3D model "Bottle" and "Balloon vehicle"

The two main tasks in specifying local semantics of model parts are segmentation and annotation. Many methods employing geometric criteria for model segmentation were proposed in the last decade, including Region growing (Pavlidis and Liow, 1990), Fitting primitives (Attene et al., 2006), Random walks (Lai et al., 2008), Random cuts (Golovinskiy and Funkhouser, 2008), Reeb graph (Biasotti et al., 2008)) and Shape diameter (Shapira et al., 2008). A recent survey can be found in (Chen et al., 2009). These methods make use of curvature, normal direction, geodesic distance or volume for segmentation. However, approaches using pure geometric attributes hardly produce meaningful model parts for annotation (Attene et al., 2009). Therefore, such methods usually need manually tuned parameters to get satisfactory and consistent results. Once 3D models have been segmented, some 
Interactive phase

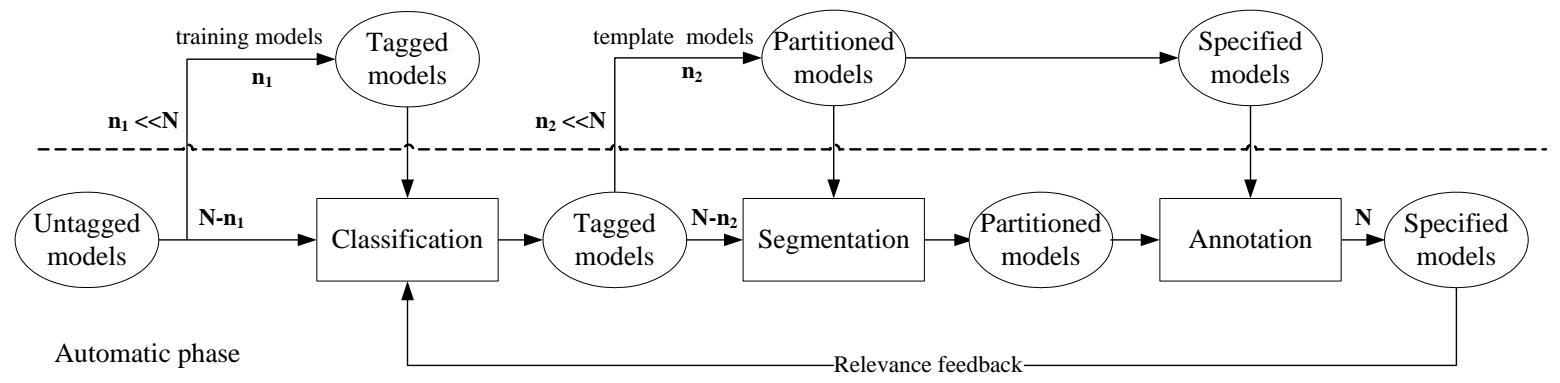

Fig. 2. The framework of our method for specifying semantics of 3D models

methods (Attene et al., 2009 and Robbiano et al., 2007), use well-defined tag dictionaries to annotate model parts manually. The papers about these methods also point out that automatic annotation of model parts could be done by exploiting topologic and geometric properties of parts, but they do not give a concrete way for such automatic annotation.

Recently, a few approaches combined 3D model segmentation and labeling with recognition of model shape; they are inspired by image segmentation in computer vision (Tu et al., 2005, and Schnitman et al., 2006). One such method (Golovingskiy and Funkhouser, 2009) simultaneously segments 3D models by matching points among a set of models. This method can segment 3D models in a class consistently, and transfer labels based on matches. However, the method needs all 3D models to be classified first, and it is not suitable for large sets of 3D models: it needs to consider each pair of models for correspondences. Another method (Kalogerakis et al., 2010) learns a large variety of geometric and contextual label features from a collection of labeled training models, and uses these label features to segment models and label model parts automatically. This method achieved a significant improvement compared with current segmentation methods. However, it requires consistently labeled models for training, and the performance typically drops with fewer training models, because it combines multiple geometric attributes and needs to determine which attributes are distinguished for certain types of models in the learning process. Our method to specify semantics of 3D models also combines segmentation with recognition. Compared with method (Kalogerakis et al., 2010), our method needs much less training models because we rely on subgraph matching to annotate model parts. Another advantage of our method is that the framework can easily incorporate other, better segmentation methods, and thus improve the results of specification of semantics.

\section{FRAMEWORK OF THE PROPOSED METHOD}

First, we refer to 3D models without any semantics as untagged models. Once models have been classified into a particular class we call them tagged models, after the segmentation we call them partitioned models, and once their parts have been annotated we call them specified models. The specified models will be further used as tagged models and improve the classification by relevance feedback. Every relevance feedback step is an iteration of the classification. This leads to three major steps in the method: classification, segmentation and annotation.

The workflow of the system is illustrated in Fig. 2. It also shows the breakup between the automatic phase and the interactive phase: for each step, the user needs to interactively create some training data that will be used in the automatic phase. For example, the user can classify one table model, after which the method will classify all other table models in the same class, possibly aided by the relevance feedback. This will be further explained in the next subsections.

\subsection{Model classification step}

In this section, we use the most popular method $\mathrm{SH}$ (Saupe and Vranic, 2011) to compute the similarity between models and SVM to classify the 3D models due to its robustness and simplicity. In order to use SVM to classify the models, we need to choose appropriate training models. Training models have a large impact on the classification result. Less training models would result in a bad classification result. However, too many training models need lots of tedious work and are not suitable for large sets of 
3D models. The best training models should spread over all the ranges of 3D models.

Therefore, we rely on the k-means method to cluster all the 3D models and choose several models from each class. We assign tags from the semantic library (Tutenel et al., 2009) to these models manually, and use these tagged models as training models. Then, we classify all the remaining untagged models based on the training models and associate these untagged models with the tag of the tagged models in the same class. In the end, the untagged models are specified. The specified models are further used to improve the classification.

\subsection{Model segmentation step}

Since 3D model segmentation is out of the scope of this paper, we rely on the currently mature methods for model segmentation. 3D models can be generally divided into two categories: man-made models, having obvious boundaries, and freeform models. Man-made models are much easier segmented compared with freeform ones. For example, we could simply use Region growing (Pavlidis and Liow, 1990) to segment a man-made model into several different parts automatically. However, this method fails to work on freeform models. We have to use other, more sophisticated methods, such as SDF (Shapira et al., 2008), to cope with such freeform models. This method usually provides users with an interactive way of manipulating parameters to get a correct segmentation result.

We argue that, with the global semantics associated to 3D models, we can reduce the users' interaction for segmentation since models with the same tag may have the same segmentation method and even have similar parameters of the same method. For each group of models with the same tag, we choose only a few template models and segment them interactively. We use the segmentation method (and parameters) of the template models to partition the rest of the models in the same group automatically.

Choosing the training models wisely is obviously an important factor towards good specification results. When there are a number of different model shapes in the same class, the training models should all be models of the same shape, but instead reflect the variability of the shapes. A simple example might be the table class. A set of models might contain a number of round tables and a number of rectangular tables. For the best result, both round and rectangular tables need to be chosen as training models to guarantee optimal results.

Choosing the training models wisely is obviously an important factor towards good specification results. If there are a number of different model shapes in the same class, the training models should reflect the variability of the shapes. A simple example might be the table class. A set of models might contain a number of round tables and a number of rectangular tables. For the best result, both round and rectangular tables need to be chosen as training models.

\subsection{Model annotation step}

Once a template model has been segmented, we annotate some model parts based on the tags from the semantic library (Tutenel et al., 2009). An AAG is defined to represent the annotated model parts, where each node denotes a model part and an edge keeps the relationships between two model parts. The automatically partitioned models are also represented by AAGs. We refer to the AAGs constructed from the template models as sub-AAGs because not all the parts of the template model are annotated. Therefore, the automatic annotation can be accomplished by subgraph matching, finding a mapping between a sub-AAG and an AAG. The details of this part are further described in the next section.

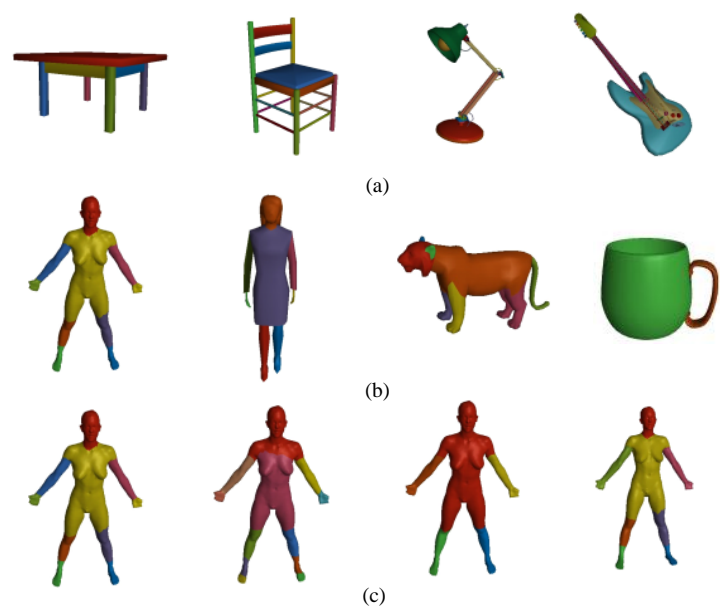

Fig. 3. Segmentation results (a) segmentation of man-made models (b) segmentation of freeform models (c) different parameters of segmentation for a freeform model

\section{SPECIFICATION OF LOCAL SEMANTICS}

Specification of semantics for the model parts is composed of a segmentation step and an annotation step. Due to the fact that there is no single 
segmentation method suitable for all 3D models, we use multiple methods for segmentation. After the tagged models have been segmented, we resort to AAGs to represent the partitioned models, and use subgraph matching to annotate model parts automatically.

\subsection{Multiple segmentation methods}

Model segmentation is a difficult field in computer graphics. To our knowledge, there is no one method that fits all types of 3D models. Therefore, we rely on two mature methods for segmentation in our system, since the segmentation of man-made models and freeform models are quite different. For example, some man-made models (Fig. 3 (a)), can be automatically segmented into different model parts by Region growing (Pavlidis and Liow, 1990). However, this method fails to work on freeform models (Fig. 3 (b)). We have to make use of SDF (Shapira et al., 2008) to segment these freeform models. Even with the SDF method, a 3D model can be partitioned quite differently with different parameters (Fig. 3 (c)). Therefore, we need to determine the appropriate segmentation method and parameters from the training models interactively, and use this method and these parameters for segmenting other models in the same class.

\subsection{AAG construction}

After all the models have been segmented, we use AAGs to represent the partitioned models. Suppose a partitioned 3D model is $M=\left\{m_{1}, m_{2}, \cdots, m_{n}\right\}$. It can be represented by an AAG $G(V, E)$, where each segmented part $m_{i}$ is denoted as a node $v_{i} \in V$; the graph edge $e\left(m_{i}, m_{j}\right) \in E$ exists whenever the two parts $m_{i}, m_{j} \in M$ are adjacent. The node $v_{i}$ keeps the geometric attributes of part $m_{i}$, such as its volume, surface area, main axis and number of adjacent parts. The edge $e\left(m_{i}, m_{j}\right)$ keeps the relationships between parts $m_{i}$ and $m_{j}$, such as distance and relative position (parallel or perpendicular). Two partitioned 3D models and their related AAGs are shown in Fig. 4.

In our system, we compute the geometric attributes and relationships of the bounding box of a part, rather than of the part itself, because of efficiency and robustness. It is much faster to compute the volumes and adjacency relationships of bounding boxes than of the actual model parts. Most algorithms for calculating the volume also fail to cope with models with holes. In our experiments, using the bounding box instead of the part itself proved sufficient, despite the slightly decreased precision.

The geometric attributes and relationships kept in nodes and edges of an AAG are critical for subgraph matching. Therefore, we need to consider the attributes and relationships which are invariant when the models are scaled, translated or rotated, and even when some pose changes, such as a human model in standing and walking poses. Volume is an important attribute. A node with a large volume is unlikely to match a node with a small volume. However, the volume of a model part is not scale invariant, and thus it needs to be normalized before using. Adjacency relationships of model parts are also very important for matching. In our experiments, we consider three cases: disconnection, connection and containment. If the bounding boxes of two model parts do not intersect, the adjacent relationship is disconnection. If the bounding boxes intersect but do not contain each other, the relationship is connection; otherwise, the relationship is containment. Parallel or perpendicular structures of models also help the procedure of subgraph matching. We need to keep these relationships in the AAG.

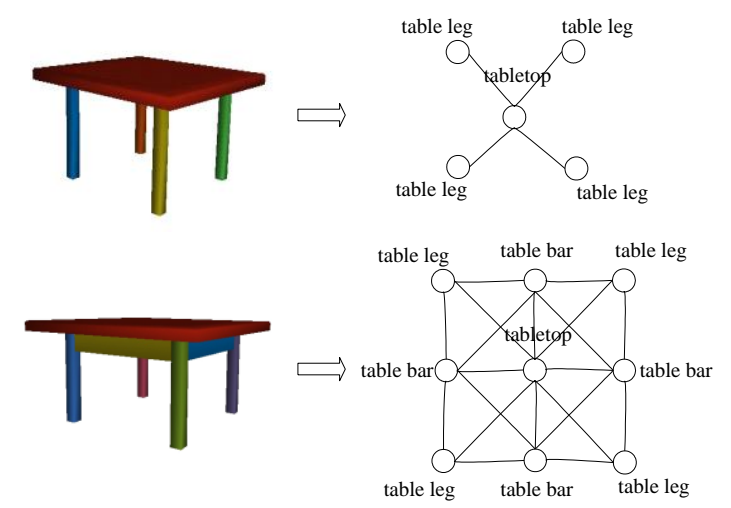

Fig. 4. Segmented models are represented by AAG

\subsection{Subgraph matching}

We annotate the parts of template models interactively, and keep the annotation in the nodes of their sub-AAGs. Therefore, the automatic annotation of the rest of the models could be done by subgraph matching, which finds a one-to-one mapping between nodes of a sub-AAG and an AAG.

Subgraph matching is an NP-complete problem. The time requirements of brute force matching algorithms increase exponentially with the size of the input graphs. There are several available algorithms, such as Ullmann's method (Ullmann, 1976), SD (Schmidt and Druffel, 1976), and VF 
(Cordella et al., 2001). Ullmann proposed a depthfirst search-based algorithm with refinement procedures, which is now the most popular and frequently used algorithm for subgraph isomorphism. Our system also makes use of Ullmann's method for AAG subgraph matching and automatic annotation. For example, in Fig. 5, if there is a correspondence between a sub-AAG and an AAG, we will annotate the related nodes of the AAG as a sub-AAG.

sub-AAG

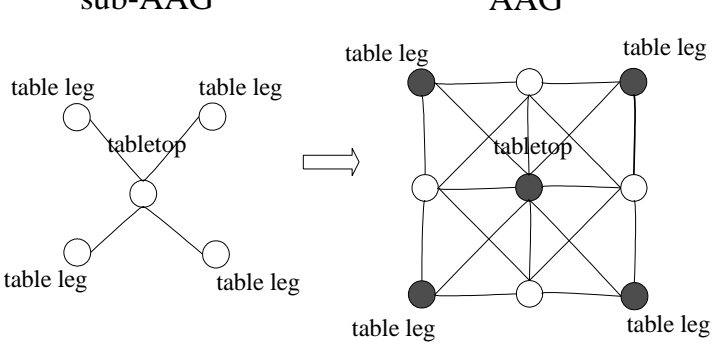

Fig. 5. Subgraph matching

In the refinement procedures of Ullmann's method, the core part is to determine whether a node $v_{n}$ of the sub-AAG corresponds to a node $v_{n}^{\prime}$ of the AAG. Suppose we have found a mapping between $G_{1}=\left\{v_{1}, v_{2}, \cdots, v_{n-1}\right\}$ from the sub-AAG and $G_{2}=\left\{v_{1}^{\prime}, v_{2}^{\prime}, \cdots, v_{n-1}^{\prime}\right\}$ from the AAG, i.e., these are isomorphic, then we need to determine whether the next node $v_{n}$ corresponds to $v_{n}^{\prime}$ or not. We use several criteria to judge this, e.g., we consider the geometric attributes of nodes and relationships between them. The pseudo code is in Algorithm 1:

\footnotetext{
Algorithm 1. Verify the paired nodes Bool VerifyNextNodes $\left(G_{1}, G_{2}, v_{n}, v_{n}^{\prime}\right)$ //Initialization

$G_{1}=\left\{v_{1}, v_{2}, \cdots, v_{n-1}\right\}, G_{2}=\left\{v_{1}^{\prime}, v_{2}^{\prime}, \cdots, v_{n-1}^{\prime}\right\}$

for each $v_{i} \in G_{1}, v_{i}^{\prime} \in G_{2}, i=1, \cdots, n-1$

//Begin to match

if the adjacency relationship of $v_{i}$ and $v_{n}$ is not equal to that of $v_{i}^{\prime}$ and $v_{n}^{\prime}$

$$
\text { return false }
$$
}

if the volume ratio of $v_{i}$ and $v_{n}$ is not close to the volume ratio of $v_{i}^{\prime}$ and $v_{n}^{\prime}$

$$
\text { return false }
$$

if $v_{i}$ is parallel (perpendicular) to $v_{n}$

if $v_{i}^{\prime}$ is not parallel(perpendicular) to $v_{n}^{\prime}$ return false

\section{//Otherwise}

return true

To further optimize the above algorithm, we take advantage of prior knowledge to reduce the search space. The details of this are described in Section 5.4. Meanwhile, the number of model parts is never really large: less than 20 in most of our experiments, and never more than 100 in practice. Therefore, the execution time of the algorithm remains acceptable although the subgraph matching algorithm is an NPcomplete problem.

\section{PROTOTYPE SYSTEM}

We will now describe the workflow of our implemented prototype system. We will discuss where and how the user needs to intervene in the process, and how the automatic steps (classification, segmentation and annotation) work.

\subsection{User interaction}

User interaction serves two tasks: specify global semantics and local semantics of a few models interactively. For specifying global semantics, we need to specify the number of clusters and choose the training models for SVM classification. Once all 3D models have been classified, we choose a few template models from each class and specify their local semantics, including interactive segmentation and annotation.

We use a semantic library (Tutenel et al., 2009) to annotate models and model parts. The aim of the semantic library is to provide a set of semantic classes for each 3D model. A semantic class describes detailed information about 3D models associated to that class, ranging from class names and physical properties, to relationships with other classes (and therefore their associated models). For example, in Fig. 6, the class "Table" consists of five parts (4 instances of the "Table leg" class and 1 of the "Table top" class) and inherits from the parent class "Furniture".

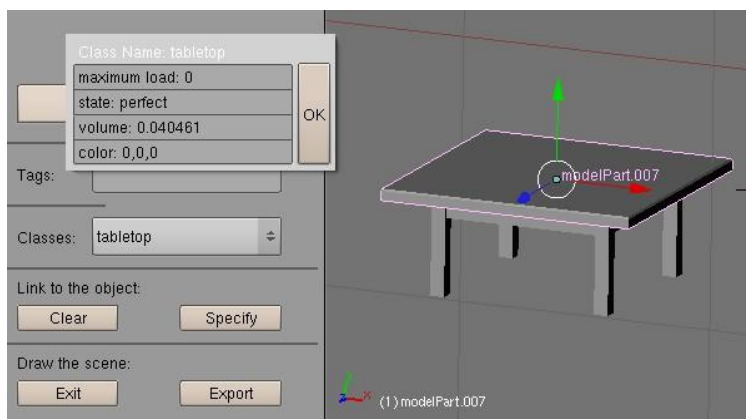

Fig. 6. Specification of semantics of 3D models 


\subsection{Automatic classification}

We use SVM for classification due to its robustness and simplicity. The RBF kernel function is usually the first choice for SVM. This kernel function can handle nonlinear problems by increasing the dimensionality of space. However, in this work, we use a linear kernel instead of RBF because the dimensionality of the shape descriptor is large (1024) (Hsu et al., 2003). In the example of Fig. 7, the model with the red box is the training model, which is accomplished as described in Section 5.1. The rest of the models in Fig.7 are considered to be similar to the training model by SVM. The classification results are almost consistent with human perception, except the last model, the "Table" model, which is wrongly classified into the group.

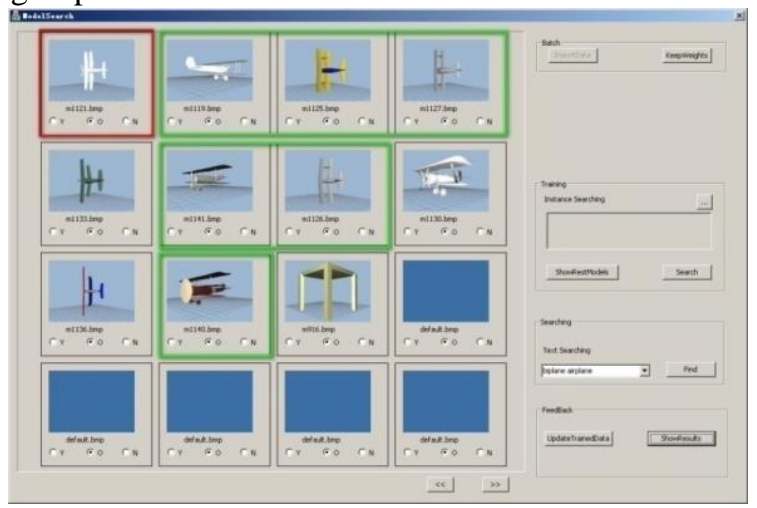

Fig. 7. Automatic Classification using training data

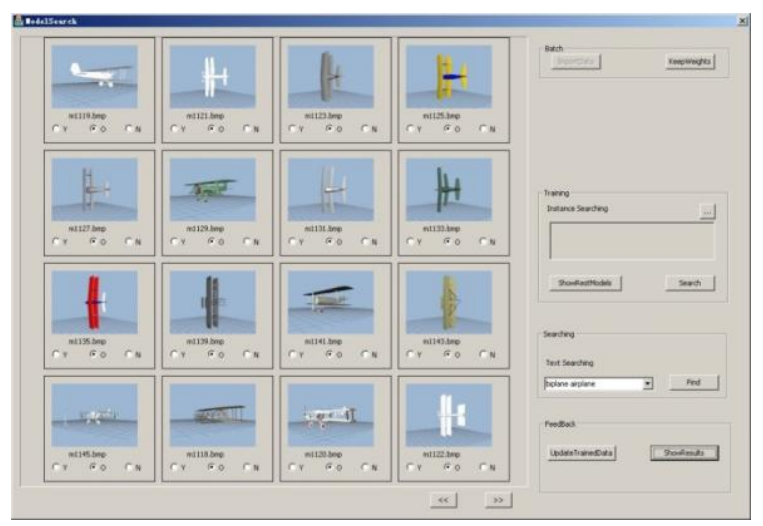

Fig. 8. The classification result after one iteration

Only a part of similar models are classified into a group by SVM with the limited training models because we expect to reduce user interaction. Some models, which belong to different classes, also end up in the same group due to the semantic gap. The relevance feedback can be used to improve the classification result. The specified models in the end are used as reference models to refine the classification hyperplane of SVM. For example, in Fig. 7, the models in the green box are specified models. Fig. 8 shows the classification results after using the new training models. The results improved quite a lot compared with Fig. 7.

\subsection{Automatic segmentation}

Once all the tagged models have been classified, we rely on template models segmented interactively in Section 5.1 to partition the other tagged models automatically. For each class of tagged models, we use the same segmentation method (and parameters) of template models for segmentation. For example, in Fig. 9 (a), the template model is segmented by region growing (Pavlidis and Liow, 1990) and the rest of the models as well. In Fig. 9 (b), the template model is segmented by SDF (Shapira et al., 2008). We need to further consider the value of parameters of the SDF method. We use the average value of parameters in the experiments if there are multiple template models in the class. However, the SDF method with the same parameters cannot guarantee consistent segmentation even when the tagged model is quite similar to the template model.

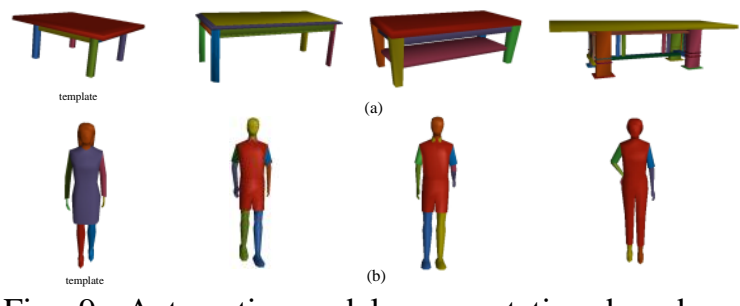

Fig. 9. Automatic model segmentation based on template models

\subsection{Automatic annotation}

The partitioned models are represented by an AAG, and subgraph matching is used for automatic annotation. Before subgraph matching, the nodes of each AAG need to be sorted according to the volume. It is appropriate to assume that nodes with a large volume are more meaningful than the ones with a small volume. Therefore, we will first search the nodes with large volume in the procedure of subgraph matching, which reduces the time complexity and ambiguity if there are multiple mappings between a sub-AAG and an AAG. 


\begin{tabular}{|c|c|c|c|c|c|c|c|c|c|c|c|c|c|c|c|c|}
\hline \multirow[t]{2}{*}{$\begin{array}{l}\text { Class } \\
\text { name }\end{array}$} & \multirow[t]{2}{*}{$\begin{array}{c}3 \mathrm{D} \\
\text { models } \\
(\mathrm{m})\end{array}$} & \multicolumn{3}{|c|}{$\begin{array}{l}\text { Classified } \\
\text { models (c) }\end{array}$} & \multicolumn{3}{|c|}{$\begin{array}{l}\text { Specified } \\
\text { models }(n)\end{array}$} & \multicolumn{3}{|c|}{$\begin{array}{c}\text { Global } \\
\text { specification rate } \\
(\%)\end{array}$} & \multicolumn{3}{|c|}{$\begin{array}{l}\text { Local specification } \\
\text { rate }(\%)\end{array}$} & \multicolumn{2}{|c|}{$\begin{array}{l}\text { Average } \\
\text { specification } \\
(\%)\end{array}$} & \multirow{2}{*}{$\begin{array}{c}\text { rate } \\
3\end{array}$} \\
\hline & & 1 & 2 & 3 & 1 & 2 & 3 & 1 & 2 & 3 & 1 & 2 & 3 & 1 & 2 & \\
\hline $\begin{array}{c}\text { seat } \\
\text { furniture }\end{array}$ & 77 & 26 & 37 & 38 & 22 & 31 & 32 & 33.8 & 48.1 & 49.4 & 84.6 & 83.8 & 84.2 & 28.6 & 40.3 & 41.6 \\
\hline $\begin{array}{l}\text { table } \\
\text { furniture }\end{array}$ & 78 & 24 & 43 & 47 & 21 & 37 & 41 & 30.8 & 55.1 & 60.3 & 87.5 & 86.0 & 87.2 & 26.9 & 47.4 & 52.6 \\
\hline $\begin{array}{c}\text { car } \\
\text { vehicle }\end{array}$ & 113 & 54 & 75 & 80 & 52 & 72 & 77 & 47.8 & 66.4 & 70.8 & 96.3 & 96.0 & 96.3 & 46.0 & 63.7 & 68.1 \\
\hline $\begin{array}{l}\text { human } \\
\text { biped }\end{array}$ & 149 & $\begin{array}{l}11 \\
5\end{array}$ & 130 & 134 & 76 & 85 & 87 & 77.2 & 87.2 & 89.9 & 66.1 & 65.4 & 65.7 & 51.0 & 57.1 & 59.1 \\
\hline $\begin{array}{l}\text { winged } \\
\text { vehicle }\end{array}$ & 242 & 139 & 171 & 171 & 101 & 124 & 124 & 57.4 & 70.7 & 70.7 & 72.7 & 72.5 & 72.5 & 41.7 & 51.2 & 51.2 \\
\hline & & & verag & & & & & 49.4 & 65.5 & 68.2 & 81.4 & 80.7 & 81.2 & 38.9 & 51.9 & 54.5 \\
\hline
\end{tabular}

Table 1: Results of automatic specifying semantics of 3D models

\section{RESULTS}

We used 3D models from the PSB (Shilane et al., 2004) to validate the framework proposed in this paper. The database of PSB is classified with 4 resolutions, ranging from coarse level to detailed level. The more detailed the level is, the more categories, and the fewer models are contained in each category. Since our framework is aimed at specifying semantics of large sets of models, we prefer to have sufficient models in each class. Therefore, we chose "coarse1" level (1814 models are divided into 38 categories) in our experiments.

We used global specification rate $s r_{\text {global }}$, local specification rate $s r_{\text {local }}$ and average specification rate $s r_{\text {ave }}$ as indicators for the performance of our prototype system. These indicators are defined as follows:

$$
\begin{gathered}
s r_{\text {global }}=\frac{c}{m}, \\
s r_{\text {local }}=\frac{n}{c}, \\
s r_{\text {ave }}=s r_{\text {global }} \times s r_{\text {local }}=\frac{n}{m}
\end{gathered}
$$

where $m$ is the number of models in a given category in PSB, $c$ is the number of models automatically classified by SVM, and $n$ is the number of automatically specified models. The global specification rate indicates the automatically classified models in the database, the local specification rate indicates the automatically specified models which are relative to classified models, and the average specification rate indicates the automatically specified models which are relative to models in the database.

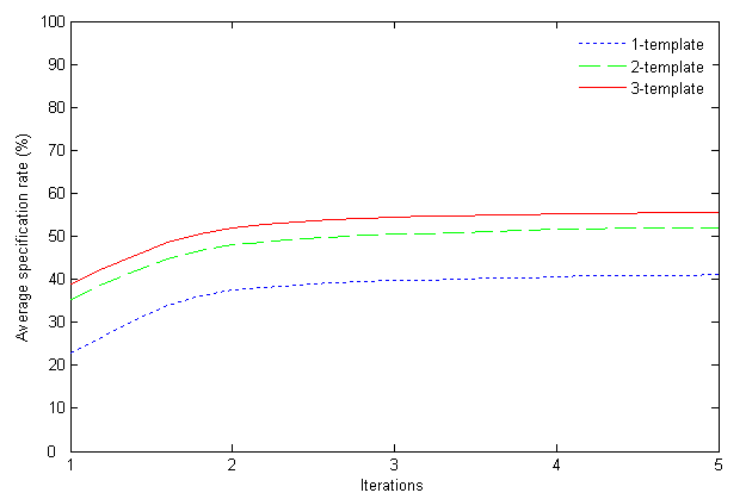

Fig. 10. Average specification rate with increasing number of template models and iterations

We set the number of categories to be 38 for clustering and chose 4 tagged models of each category as training models for SVM classification. Once all the models of the PSB were classified, we segmented and annotated 3 models of each class interactively, and used these specified models as template models for automatic segmentation and annotation. Table 1 illustrates the results of 5 major categories in the PSB with three iterations. Once the 3D models have been classified by SVM, more than $80 \%$ (local specification rate) of the models can be segmented and annotated automatically. However, the SVM classifier cannot find all the 3D models 
with the limited training models, because a class of PSB usually contains several subclasses. Therefore, the final average specification rate is only nearly $55 \%$. An impression of the impact of relevance feedback and template models is shown in Fig. 10. The average specification rate can be improved, although only to a limited extent, by increasing the number of template models and iterations. We usually need at least 2 template models of each group and 2 iterations to get a good performance of automatic annotation of models.

\section{CONCLUSIONS}

This paper presents a method for specifying semantics of 3D models with limited interaction. All the models in the database are first classified. For each class, we segment and annotate a few template models interactively. The rest of the models can be separated based on segmentation methods (and parameters) of template models. Automatic annotation can be achieved by subgraph matching which finds a map between a sub-AAG representing the parts of the template model and an AAG representing the parts of partitioned models. In the end, the specified models are used to improve the classification.

Our method can achieve a good rate of automatic annotation of 3D models with limited user interaction, especially for the 3D models that can be separated into consistent parts. Man-made models compared with freeform ones are easier to be segmented into consistent parts because the segmentation method has no parameters. Thus, manmade models have a higher specification rate, which is indicated by the average specification rate in Fig. 10.

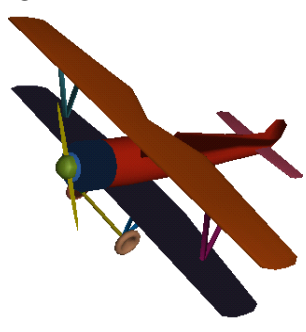

(a)

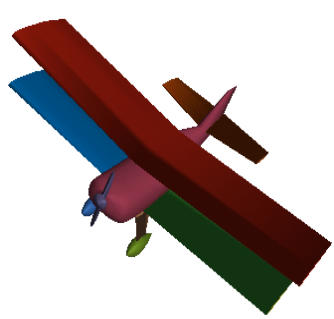

(b)
Fig. 11. Inconsistent segmentation results using the same segmentation method

However, in experiments, although we use the same segmentation method (and parameters) to segment similar models, sometimes we still get inconsistent segmentation results which means the model parts cannot be annotated successfully. In this case, we can provide more template models of each class for automatic segmentation and annotation. For example, in Fig. 11, the two models from the class "winged vehicle" are partitioned inconsistently (one of the wings is segmented into two parts) with the same segmentation algorithm. We cannot use the template model (a) to annotate model (b). Therefore, we also use model (b) as a template model for automatic annotation of the class "winged vehicle".

In future work, we will test more segmentation methods, especially for freeform models. We will also consider more geometric and topologic relationships among segments in the procedure of subgraph matching for automatic annotation.

\section{ACKNOWLEDGEMENTS}

The work of Xin Zhang has been supported by Netherlands Organization for International Cooperation in Higher Education (Nuffic). This research has also been partly supported by the GATE project, funded by the Netherlands Organization for Scientific Research (NWO).

\section{REFERENCES}

Attene M., Falcidieno B., Spagnuolo M. (2006). 'Hierarchical mesh segmentation based on fitting primitives', The Visual Computer, vol. 22(3), pp. 181 $-193$.

Attene M., Robbiano F., Spagnuolo M., Falcidieno B. (2009). 'Characterization of 3D shape parts for semantic annotation', Computer-Aided Design, vol. 41(10), pp. $756-763$.

Biasotti S., Giorgi D., Spagnuolo M., Falcidieno B. (2008). 'Reeb graphs for shape analysis and application', Theorerical Computer Science, vol. 392(1-3), pp. $5-22$.

Bronsvoort W.F., Bidarra R., van der Meiden H.A., Tutenel T. (2010). 'The increasing role of semantics in object modeling', Computer-Aided Design and Applications, vol. 7(3), pp. $431-440$.

Carpenter W.C. and Hoffman M.E. (1997). 'Selecting the architecture of a class of back-propagation neural network used as approximators', Artificial Intelligence for Engineering Design, vol. 11, pp. 33 - 44.

Chen X., Golovinskiy A., Funkhouser T. (2009). 'A benchmark for 3D mesh segmentation', TOG: Proceedings of ACM SIGGRAPH, vol. 28(3), a.73.

Cordella L.P., Forggia P., Sansone C., Vento M. (2001). 'An improved algorithm for matching large graphs', Proceedings of the 3rd International Association for Pattern Recognition Workshop on Graph-Based Representation in Pattern Recognition, Ischia, Italy, pp. $149-159$. 
Giacinto G. and Roli F. (2004). 'Bayesian relevance feedback for content-based image retrieval', Pattern Recognition, vol. 37(7), pp. 1499-1508.

Golovinskiy A. and Funkhouser T. (2008). 'Randomized cuts for 3D mesh analysis', Proceedings of ACM SIGGRAPH Asia, vol. 27(8), a.145.

Golovingskiy A. and Funkhouser T. (2009). 'Consistent segmentation of 3D models', Computers \& Graphics, vol. 33(3), pp. $262-269$.

Han E.H. and Karypis G. (2000). 'Centroid-based document classification: analysis and experimental results', Proceeding of 4th European Conference on Principles of Data Mining and Knowledge Discovery, Lyon, France, pp. $424-431$.

Hsu C.W., Chang C.C., Lin C.J. (2003). 'A practical guide to support vector classification', Technical report, Department of Computer Science, National Taiwan University.

Kalogerakis E., Hertzmann A., Singh K. (2010). 'Learning $3 \mathrm{D}$ mesh segmentation and labeling', TOG: Proceedings of ACM SIGGRAPH, vol. 29(4), pp. a.102.

Kessing J., Tutenel T., Bidarra R. (2009). 'Services in game worlds: a semantic approach to improve object interaction', Proceedings of the International Conference on Entertainment Computing, ICEC 2009, Paris, France, pp. 276 - 281.

Lai Y.K., Hu S.M., Martin R.R., Rosin P.L. (2008). 'Fast mesh segmentation using random walks', Proceedings of the ACM symposium on Solid and Physical Modeling, New York, U.S.A., pp. 183 - 191.

Novotni M. and Klein R. (2004). 'Shape retrieval using 3D Zernike descriptors', Computer-Aided Design, vol. 36(11), pp. 1047 - 1062.

Novotni M., Park G.J., Wessel R., Klein R. (2005). 'Evaluation of kernel based methods for relevance feedback in shape retrieval', Proceeding of the 4th International Workshop on Content-Based Multimedia indexing (CBMI), Riga, Latvia.

Osada R., Funkhouser T., Chazelle B., Dobkin D. (2002). 'Shape distributions', ACM Transactions on Graphics, vol. 21(4), pp. $807-832$.

Pavlidis T. and Liow Y.T. (1990). 'Integrating region growing and edge detection', IEEE Transactions on Pattern Analysis and Machine Intelligence, vol. 12(3), pp. $225-233$.

Robbiano F., Attene M., Spagnuolo M. (2007). 'Partbased annotation of virtual 3D shapes', Proceedings of the International Conference on Cyberworlds, Washington D.C., U.S.A., pp. 427 - 436.

Rocchio J.J. (1971). 'Relevance feedback in information retrieval', the SMART Retrieval System: Experiments in Automatic Document Processing, pp. 313 - 323.

Saupe D. and Vranic D.V. (2001). '3D model retrieval with spherical harmonics and moments', Proceedings of the $23^{r d}$ DAGM-Symposium, Munich, Germany, pp. $392-397$.

Schmidt D.C. and Druffel L.E. (1976). 'A fast backtracking algorithm to test directed graphs for isomorphism using distance matrices', Journal of ACM, vol. 23(3), pp. 433 - 445.

Schnitman Y., Caspi Y., Cohen-Or D., Lischinski D. (2006). 'Inducing semantic segmentation from an example', Proceedings of Computer Vision - ACCV, Hyderabad, India, pp. $374-384$.

Shapira L., Shamir A., Cohen-Or D. (2008). 'Consistent mesh partitioning and skeletonisation using the shape diameter function', The Visual Computer, vol. 24(4), pp. $249-259$.

Shilane P., Min P., Kazhdan M., Funkhouser T. (2004). 'The Princeton shape benchmark', Proceedings of Shape Modeling International (SMI), Genova, Italy, pp. $167-178$.

Smeulders A.W.M., Worring M., Santini S., Gupta A., Jain R. (2000). 'Content-based image retrieval at the end of the early years', IEEE Transactions on Pattern Analysis and Machine Intelligence, vol. 22(12), pp. $1349-1380$

Tangelder J.W.H. and Veltkamp R.C. (2004). 'A survey of content based 3D shape retrieval methods', Proceedings of International Conference on Shape Modeling and Applications, Palazzo Ducale, Genova, Italy, pp. $145-156$.

Tu Z., Chen X., Yuille A.L., Zhu S. (2005). 'Image parsing: unifying segmentation, detection and recognition', International Journal of Computer Vision, vol. 63(2), pp. $113-140$.

Tutenel T., Bidarra R., Smelik R.M., de Kraker K.J. (2008). 'The role of semantics in games and simulation', ACM Computer in Entertainment, vol. 6(4), pp. $1-35$.

Tutenel T., Bidarra R., Smelik R.M., de Kraker K.J. (2009). 'Rule-based layout solving and its application to procedural interior generation', Proceedings of the CASA'09 Workshop on 3D Advanced Media in Gaming and Simulation, Amsterdam, the Netherlands, pp. $15-24$.

Ullmann J.R. (1976). 'An algorithm for subgraph isomorphism', Journal of ACM, vol. 23(1), pp. 31 42. 